\title{
ARTICLE
}

Clinical Study

\section{The efficacy of VEGFR TKI therapy after progression on immune combination therapy in metastatic renal cell carcinoma}

\author{
Pedro Coelho Barata', Alfonso Gomez De Liano², Prateek Mendiratta', Valerie Crolley², Bernadett Szabados², Laura Morrison², \\ Laura Wood ${ }^{1}$, Kimberly Allman ${ }^{1}$, Allison Tyler ${ }^{1}$, Allison Martin ${ }^{1}$, Timothy Gilligan ${ }^{1}$, Petros Grivas ${ }^{1}$, Moshe Ornstein ${ }^{1}$, Jorge A. Garcia ${ }^{1}$, \\ Thomas Powles ${ }^{2}$ and Brian I. Rini ${ }^{1}$
}

\begin{abstract}
BACKGROUND: The outcome of patients who progress on front-line immune-based combination regimens (IC) including immune checkpoint inhibitors (CPI) and receive subsequent systemic therapy is unknown.

METHODS: Retrospective analysis of consecutive patients with clear-cell mRCC who progressed on one of seven clinical trials investigating an IC and received $\geq 1$ line of subsequent VEGFR TKI therapy.

RESULTS: Thirty-three patients [median age 57 (37-77), 85\% male, 73\% ECOG 0] were included. For evaluable patients $(N=28)$, the best response to first subsequent therapy was $29 \%$ partial response, $54 \%$ stable disease, and $18 \%$ progressive disease. The median PFS (mPFS) for first subsequent therapy was 6.4 months ( $95 \% \mathrm{Cl}, 4.4-8.4)$; no difference in mPFS by prior type of IC (VEGFR TKI-CPI vs. CPI-CPI) was noted $(p=0.310)$. Significant AEs were observed in $30 \%$ of patients, more frequently transaminitis $(9 \%)$.

CONCLUSIONS: VEGFR TKIs have clinical activity in mRCC refractory to IC therapy, possibly impacted by the mechanism of prior combination therapy.
\end{abstract}

British Journal of Cancer (2018) 119:160-163; https://doi.org/10.1038/s41416-018-0104-z

\section{INTRODUCTION}

Medical treatment for metastatic renal cell carcinoma (mRCC) has expanded considerably from a nonspecific immune approach to targeted therapies against vascular endothelial growth factor receptor (VEGFR) and mammalian target of rapamycin (mTOR). ${ }^{1-5}$ More recently, the survival benefit and subsequent approval of the immune checkpoint inhibitor (CPI) nivolumab, for patients with refractory $\mathrm{mRCC}$ has ushered in a new era of research into potential combination approaches to improve outcomes and to potentially overcome resistance. ${ }^{6}$ Preclinical data suggesting synergy between angiogenic inhibition and the combination of anti-cytotoxic $T$ lymphocyte-associated antigen-4 with CPIs has led to the development of immune-oncology-based combination (IC) regimens. ${ }^{7,} 8$ Early-phase trials with different IC regimens resulted in promising clinical activity with high response rates and manageable tolerability in heavily treated mRCC patients. ${ }^{9-11}$ The combination of nivolumab and iplimumab resulted in higher overall response rate and prolonged overall survival (OS) compared to sunitinib in intermediate and poor-risk mRCC patients (Checkmate-214) and other phase III studies testing several ICs are ongoing thus, the front-line treatment of mRCC is likely to change. ${ }^{12}$

Unfortunately, most patients eventually progress and require additional treatment after an IC regimen. The clinical outcome of patients who receive subsequent systemic therapy is undetermined. To assess the safety and efficacy of subsequent treatments in the post-immunotherapy setting, we conducted a retrospective review of consecutive mRCC patients from two academic centres who had received a prior IC and were subsequently treated with a targeted agent.

\section{METHODS}

This was a retrospective, multi-institutional study that included patients with clear-cell mRCC enroled in one of the seven clinical trials investigating a combination regimen including a CPI (NCT02420821, NCT01984242, NCT01472081, NCT02231749, NCT02853331, NCT02493751, NCT02684006). All patients were required to have disease progression at the discretion of the treating physician and were subsequently treated with at least one VEGFR-TKI. Patients who remained on all or part of the IC regimen after coming off trial were excluded. Patients were grouped into those who had received a combination of $\mathrm{CPI}$ and VEGFR-TKI and those who had received a combination of two CPIs.

Study population

Eighty-nine mRCC patients enroled in one of the seven clinical trials investigating an IC at two institutions (Taussig Cancer Center Cleveland Clinic, Cleveland; Barts Cancer Institute, London) were retrospectively collected, in compliance with IRB guidelines. Electronic medical records were reviewed for

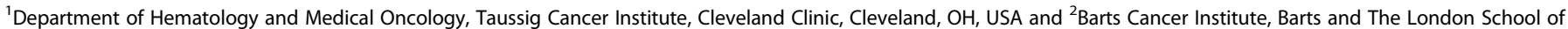
Medicine and Dentistry, Queen Mary of London, London, UK

Correspondence: Brian I. Rini (rinib2@ccf.org)

Received: 12 December 2017 Revised: 4 April 2018 Accepted: 12 April 2018

Published online: 24 May 2018 
VEGF therapy after immune combination in mRCC PC Barata et al.

baseline characteristics. Patients were categorised into three risk categories: favourable-risk, intermediate-risk and poor-risk according to The International Metastatic Renal Cell Carcinoma Database Consortium (IMDC) risk criteria. ${ }^{13}$ Outcome measures were also retrieved from chart reviews including progressionfree survival (PFS), investigator-assessed overall response rate (ORR) according to RECIST v1.1 ${ }^{14}$ and adverse events (AEs) using CTCAE v4.0. ${ }^{15}$

Statistical analysis

The primary objective of this study was to characterise ORR and PFS of mRCC patients treated with at least one subsequent therapy after progression on IC. The data reported here (including OS) was limited to protect the ongoing phase 3 trials testing ICs that have not yet been presented. PFS was defined as the time period between first subsequent treatment initiation and drug discontinuation due to progression, death, or last follow-up. Those who were still on treatment and those who discontinued therapy without disease progression were censored at the date of last evaluation. Distributions of PFS were estimated using Kaplan-Meier methodology. The statistical analysis was performed using SPSS version 23 and $p<0.05$ was considered statistically significant.

\section{RESULTS}

Baseline characteristics

Between February 2015 and September 2017, 89 patients with mRCC enroled on an IC protocol. Among the 41 patients who progressed, 8 patients were excluded: five patients remained on axitinib at an escalated dose after coming off trial with a combination regimen that included axitinib; one patient received nivolumab as first subsequent therapy after IC and two patients died before starting subsequent therapy. The final cohort included 33 patients who progressed on IC protocol. Twenty-four patients were on combo at time of PD, while four patients were on singleagent VEGFR TKI, three on CPI alone and two other had both IC agents on hold due to toxicity.

Patient characteristics are summarised in Table 1. Twenty-two patients received a VEGFR-TKI in combination with a CPI, and 11 patients had a combination of two CPIs. Thirty-two patients received IC in the front-line setting while one patient was treated with ipilumumab plus nivolumab after prior treatment with frontline sunitinib.

Outcomes of subsequent treatment after IC

The median follow-up time from the initiation of the first subsequent therapy was 13 months. All patients received one subsequent therapy (axitinib $n=16$; pazopanib $n=9$; sunitinib $n=4$; cabozantinib $n=4$ ) initiated after a median of 27 days (range, 0-2 years) after disease progression on the IC regimen. Twelve patients were treated with a second subsequent therapy and five patients were treated with $\geq 3$ subsequent lines of treatment (Table 1). The median PFS and ORR for each first subsequent therapy after progression on ICs are summarised in Table 2. For patients with available response data $(n=28)$, the overall best response to first subsequent therapy were partial response (PR) in $8(29 \%)$, stable disease (SD) in $15(54 \%)$, and progressive disease (PD) in 5 (18\%; Table 2). A higher proportion of RR was observed with pazopanib (43\%) while patients who received sunitinib had no responses and 50\% (2/4) had PD as best response. The ORR was 33\% for patients previously treated with combination of two CPIs compared with $25 \%$ for CPI plus anti-VEGFR combination $(p=0.678)$. Median PFS for the first subsequent therapy was 6.4 months $(95 \% \mathrm{Cl}, 4.4-8.4)$ with seven patients remaining on treatment. The median PFS for cabozantinib was not reached, while PFS for sunitinib was 2.9 months compared with 5.6 and 6.4 months for pazopanib and axitinib, respectively (Table 2 ). The median PFS for patients previously
Table 1. Baseline characteristics prior to first subsequent therapy

\begin{tabular}{|c|c|}
\hline Characteristics & $\%$ \\
\hline Median age & $57(37-77)$ \\
\hline \multirow[t]{2}{*}{ Gender } & Male: $85 \%$ \\
\hline & Female: $15 \%$ \\
\hline \multirow[t]{2}{*}{ PS } & 0: $73 \%$ \\
\hline & 1: $27 \%$ \\
\hline \multirow[t]{3}{*}{ IMDC risk group } & Favourable: $27 \%$ \\
\hline & Intermediate: $52 \%$ \\
\hline & Poor: $21 \%$ \\
\hline \multirow[t]{5}{*}{ Location of metastases } & Lung: $76 \%$ \\
\hline & Lymph nodes: $52 \%$ \\
\hline & Bone: $30 \%$ \\
\hline & Liver: $21 \%$ \\
\hline & Locoregional: $21 \%$ \\
\hline Prior nephrectomy & $64 \%$ \\
\hline \multirow[t]{3}{*}{ IC regimen } & $\begin{array}{l}\text { Atezolizumab/bevacizumab: } \\
64 \%\end{array}$ \\
\hline & Ipilimumab/nivolumab: $33 \%$ \\
\hline & Axitinib/avelumab: $3 \%$ \\
\hline \multirow{3}{*}{$\begin{array}{l}\text { Number of subsequent systemic } \\
\text { therapies }\end{array}$} & 1: $100 \%$ \\
\hline & $2: 36 \%$ \\
\hline & $\geq 3: 15 \%$ \\
\hline \multirow{4}{*}{$\begin{array}{l}\text { First subsequent systemic therapy } \\
(n=31)\end{array}$} & Axitinib: $48 \%$ \\
\hline & Cabozantinib $12 \%$ \\
\hline & Pazopanib: 27\% \\
\hline & Sunitinib: $12 \%$ \\
\hline \multirow{9}{*}{$\begin{array}{l}\text { Second subsequent systemic therapy } \\
(n=12)\end{array}$} & Axitinib: $25 \%$ \\
\hline & Cabozantinib: $8 \%$ \\
\hline & HIF inhibitor: $8 \%$ \\
\hline & Lenvatinib/everolimus: $8 \%$ \\
\hline & mTOR inhibitor: $8 \%$ \\
\hline & Nivolumab: $8 \%$ \\
\hline & Sorafenib: $8 \%$ \\
\hline & Sunitinib: $8 \%$ \\
\hline & Tivozanib: 8\% \\
\hline \multirow{2}{*}{$\begin{array}{l}\text { Third subsequent systemic therapy } \\
(n=5)\end{array}$} & Cabozantinib: $80 \%$ \\
\hline & Everolimus: $20 \%$ \\
\hline
\end{tabular}

treated with a combination of anti-VEGFR plus CPI was 6.2 months $(95 \% \mathrm{Cl}, 5.2-7.2)$ and 7.6 months $(95 \% \mathrm{Cl}, 3.6-11.6)$ for patients treated with a combination of two CPIs ( $p=0.310$; Fig. 1). A total of 12 patients received a second subsequent therapy after IC, with a median PFS of 4.1 months $(95 \% \mathrm{Cl}$, $0-9.2)$.

Any $\mathrm{AE}$ occurred in $73 \%$ of the patients treated with first subsequent therapy (Table 3). Overall, the most frequent AEs (any grade) were fatigue (36\%), diarrhoea (30\%) and mucositis (24\%). Significant (G3/G4) AEs were reported in 10 patients and included LFTs elevation $(n=3)$, diarrhoea $(n=2)$, mucositis $(n=2)$, fatigue $(n=1)$, hypertension $(n=1)$, low platelet count $(n=1)$, and nephrotic syndrome $(n=1)$. One patient developed G4 AE (elevated LFTs) while on pazopanib. For patients with G3/4 AEs, the median time between the last IC and the first dose of VEGFRTKI was 20 days (range, 0-64). Considering all patients with significant LFTs abnormalities $(n=3)$, two started pazopanib 1 day after stopping IC (ipilimumab/nivolumab and atezolizumab/ 
Table 2. Progression-free survival (PFS) and best response (RECIST v1.1) to first subsequent treatment in evaluable patients

\begin{tabular}{llllll}
\hline & Axitinib $(N=14)$ & Pazopanib $(N=7)$ & Cabozantinib $(N=3)$ & Sunitinib $(N=4)$ & Total cohort $(N=28)$ \\
\hline Median PFS, months (Cl 95\%) & $6.4^{\mathrm{a}}(4.7-8.1)$ & $5.6(1.2-10.0)$ & $\mathrm{NR}$ & $2.9(0.0-7.6)$ & $6.4^{\mathrm{a}}(4.4-8.4)$ \\
Objective response rate (ORR) & $29 \%(4)$ & $43 \%(3)$ & $33 \%(1)$ & $0 \%(0)$ & $29 \%(8)$ \\
Stable disease (SD) & $64 \%(9)$ & $29 \%(2)$ & $66 \%(2)$ & $50 \%(2)$ & $54 \%(15)$ \\
Progressive disease (PD) & $7 \%(1)$ & $29 \%(2)$ & $0 \%(0)$ & $50 \%(2)$ & $18 \%(5)$ \\
\hline
\end{tabular}

$N R$ not reached. ${ }^{a}$ One patient who received axitinib in the third-line setting was excluded

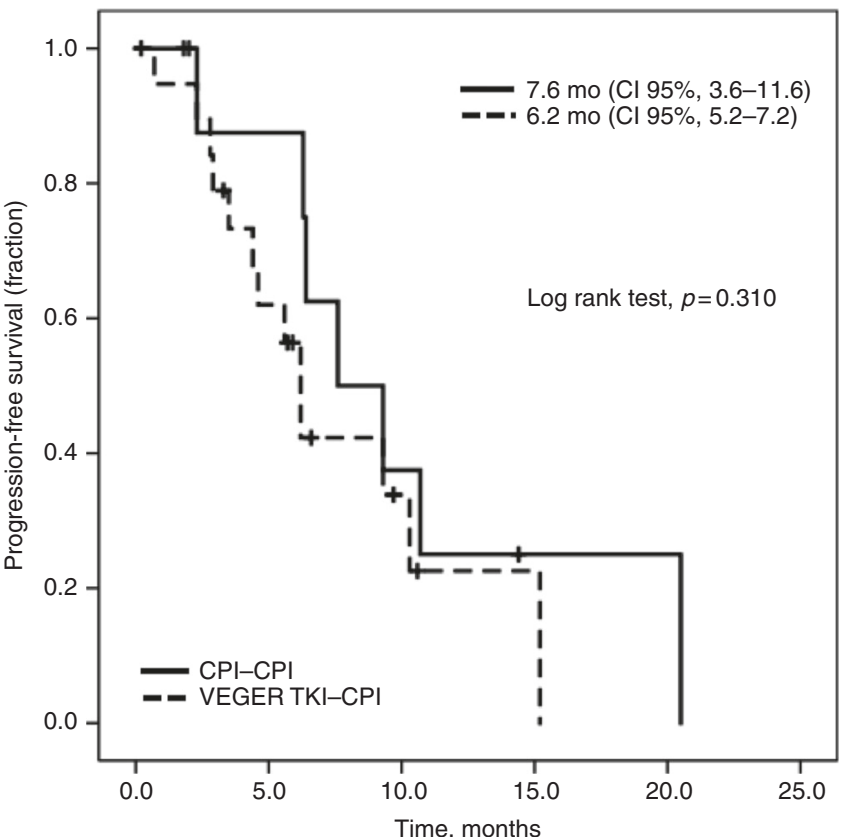

Fig. 1 Progression-free survival (PFS) curve by type of IC. mPFS median progression-free survival, mo months, $C /$ confidence interval, $C P I$ checkpoint inhibitor, IC immune-based combination

\begin{tabular}{|lllll|}
\hline $\begin{array}{l}\text { Table 3. Grade 2-4 adverse events of any cause during first } \\
\text { subsequent TKI }\end{array}$ & \multicolumn{4}{l|}{} \\
\hline Factor & Grade 2 & Grade 3 & Grade 4 & Total \\
& $2(6)$ & $0(0)$ & $0(0)$ & $2(6)$ \\
\hline Anorexia & $5(15)$ & $2(6)$ & $0(0)$ & $7(21)$ \\
Diarrhoea & $6(18)$ & $1(3)$ & $0(0)$ & $7(21)$ \\
Fatigue & $1(3)$ & $0(0)$ & $0(0)$ & $1(3)$ \\
Hand foot syndrome & $2(6)$ & $1(3)$ & $0(0)$ & $3(9)$ \\
Hypertension & $2(6)$ & $0(0)$ & $0(0)$ & $2(6)$ \\
Hypothyroidism & $5(15)$ & $2(6)$ & $0(0)$ & $7(21)$ \\
Mucositis & $2(6)$ & $0(0)$ & $0(0)$ & $2(6)$ \\
Nausea & $0(0)$ & $1(3)$ & $0(0)$ & $1(3)$ \\
Nephrotic syndrome & $1(3)$ & $0(0)$ & $0(0)$ & $1(3)$ \\
Vomiting & 0() & $2(6)$ & $1(3)$ & $3(9)$ \\
AST/ALT elevation & $0(0)$ & $1(3)$ & $0(0)$ & $1(3)$ \\
Low platelets & & & & \\
\hline
\end{tabular}

bevacizumab), respectively, while one patient started axitinib 4 weeks after ipilimumab/nivolumab discontinuation. No association was found between time to first subsequent TKI and significant $\mathrm{AEs}(p=0.288)$.

\section{DISCUSSION}

This analysis evaluated the activity of subsequent treatments after failure of front-line IC regimens in $\mathrm{BRCC}$, for which there is currently a lack of data due to the rapidly evolving field. The response and PFS observed with first subsequent therapy confirmed the activity of VEGFR TKls in this setting and in line with the standard second line therapy after progression on TKI therapy. ${ }^{16,}{ }^{17}$ Of note, a numerical advantage in objective response and PFS to subsequent was observed in patients who did not receive prior anti-VEGF agents, although not statistically significant. This is likely due to the reduced activity of anti-VEGF agents when given in sequence, although larger prospective data are required.

This work supports the continued sequencing of these agents in mRCC to maximise outcomes, although differences in clinical activity among different TKls were not able to be discerned in this small, retrospective series and will require prospective investigation.

In addition, insight into potential toxicity was noted in this series including preliminary signals, such as LFT elevation. CPIs have a prolonged half-life, and thus a longer washout period is recommended to prevent interactions with subsequent therapies. As this is an area of significant risk, this transition period requires frequent laboratory and clinical assessment.

There are several limitations of this analysis, including the retrospective nature, a relatively small sample size, the diversity of TKIs in the post-IO space and short follow up. Importantly, only patients enroled in a clinical trial testing an IC were included, who are known to have favourable clinical characteristics and not necessarily fully representative of the mRCC population seen in clinical practice. ${ }^{18,}{ }^{19}$ Nevertheless, these findings show clinical activity of anti-VEGF agents with an acceptable safety signal when given after immune-based combination therapy, supporting the sequencing of these agents as a standard of care. Validation of these findings in future clinical trials is warranted, and such trials are ongoing including the ongoing phase II studies assessing the clinical activity of pazopanib (NCT03200717) or an individualised schedule of axitinib (NCT02579811) after prior CPIs.

\section{AUTHOR CONTRIBUTIONS}

B.R. and T.P. were lead co-investigators in some of the front-line trials with $I O$ combination regimens. P.B., A.L., P.M., V.C., B.R., and T.P. collected data for this project and wrote initial version of the manuscript. P.B., A.L., P.M., V.C., B.S., L.M., L.W., K.A., A. T., A.M., T.G., P.G., M.O., J.G., T.P., B.R. helped write and approved the final version of the manuscript.

\section{ADDITIONAL INFORMATION}

Conflict of interest: The authors declare that they have no conflict of interest.

Ethical approval: Patient data was collected in compliance with both Institutional guideline.

Informed consent: Informed consent was waived for this study. 
Note: This work is published under the standard license to publish agreement. After 12 months the work will become freely available and the license terms will switch to a Creative Commons Attribution 4.0 International (CC BY 4.0).

\section{REFERENCES}

1. Motzer, R. J. et al. Pazopanib versus sunitinib in metastatic renal-cell carcinoma. N. Engl. J. Med. 369, 722-731 (2013).

2. Motzer, R. J. et al. Axitinib versus sorafenib as second-line treatment for advanced renal cell carcinoma: overall survival analysis and updated results from a randomised phase 3 trial. Lancet Oncol. 14, 552-562 (2013).

3. Motzer, R. J. et al. Sunitinib versus Interferon Alfa in metastatic renal-cell carcinoma. N. Engl. J. Med. 356, 115-124 (2007).

4. Rini, B. I. et al. Bevacizumab plus interferon alfa compared with interferon alfa monotherapy in patients with metastatic renal cell carcinoma: CALGB 90206. J. Clin. Oncol. 26, 5422-5428 (2008).

5. Motzer, R. J. et al. Efficacy of everolimus in advanced renal cell carcinoma: a double-blind, randomised, placebo-controlled phase III trial. Lancet 372, 449-456 (2008).

6. Motzer, R. J. et al. Nivolumab versus everolimus in advanced renal-cell carcinoma. N. Engl. J. Med. 373, 1803-1813 (2015).

7. Oyama, T. et al. Vascular endothelial growth factor affects dendritic cell maturation through the inhibition of nuclear factor-kB activation in hemopoietic progenitor cells. J. Immunol. 160, 1224-1232 (1998).

8. Ko, J. S. et al. Sunitinib mediates reversal of myeloid-derived suppressor cell accumulation in renal cell carcinoma patients. Clin. Cancer Res. 15, 2148-2157 (2009).

9. Atkins, M. B. et al. Phase lb dose-finding study of axitinib plus pembrolizumab in treatment-naïve patients with advanced renal cell carcinoma. J. Immunother Cancer 3(S2), P353 (2015).
10. Amin, A. et al. Nivolumab (anti-PD-1; BMS-936558, ONO-4538) in combination with sunitinib or pazopanib in patients (pts) with metastatic renal cell carcinoma (mRCC). J. Clin. Oncol. 32(15_suppl), 5010-5010 (2014).

11. Sznol, M. et al. Phase Ib evaluation of MPDL3280A (anti-PDL1) in combination with bevacizumab (bev) in patients (pts) with metastatic renal cell carcinoma (mRCC). J. Clin. Oncol. 33(7_suppl), 410-410 (2015).

12. Motzer RJ, Tannir NM, McDermott DF et al (2018) Nivolumab plus Ipilimumab versus Sunitinib in Advanced Renal-Cell Carcinoma. New England Journal of Medicine 378(14), 1277-1290.

13. Heng, D. Y. et al. Prognostic factors for overall survival in patients with metastatic renal cell carcinoma treated with vascular endothelial growth factor-targeted agents: results from a large, multicenter study. J. Clin. Oncol. 27, 5794-5799 (2009).

14. Eisenhauer, E. A. et al. New response evaluation criteria in solid tumours: revised RECIST guideline (version 1.1). Eur. J. Cancer 45, 228-247 (2009).

15. US Department of Health. Common Terminology Criteria for Adverse Events (CTCAE) Version 4.0. National Cancer Institute. 2009.

16. Rini, B. I. et al. Comparative effectiveness of axitinib versus sorafenib in advanced renal cell carcinoma (AXIS): a randomised phase 3 trial. Lancet 378, 1931-1939 (2011).

17. Choueiri, T. K. et al. Cabozantinib versus everolimus in advanced renal-cell carcinoma. N. Engl. J. Med. 373, 1814-1823 (2015).

18. Arranz Arija, J. et al. 884PSPAZO2 (SOGUG): Comparative effectiveness of pazopanib in metastatic renal carcinoma ( $\mathrm{mRC}$ ): Ineligible (I) vs eligible (E) patients for clinical trials. Ann. Oncol. 28(suppl_5), (2017).

19. Wells, J. C. et al. Third-line targeted therapy in metastatic renal cell carcinoma: results from the international metastatic renal cell carcinoma database consortium. Eur. Urol. 71(2), 204-209 (2017). 